\title{
Start-Up Time of Crossing Pedestrians with Different Signal Configurations
}

\author{
V. Wickramasinghe, P.A. Wijesinghe and K.G.M. Lakmali
}

\begin{abstract}
Pedestrian start-up time is a very important factor when designing the pedestrian signal phase at a pedestrian crosswalk. Start-up time is divided into two categories as early start-up and delayed star-up time. If a pedestrian starts to move from the curb during the red light, it is termed as early start-up time whereas if the pedestrian starts moving from the curb after the green light, then it is termed as delayed start-up time. Pedestrian crossing time is the summation of the start-up time of the pedestrian and the time required to cross the road. By adding this start-up time into the pedestrian signal phase can reduce the interaction between pedestrians and vehicles, and excess delay at pedestrian crossings. From the initial study, it was observed that start-up time is greatly influenced by the signal configuration. Thus, this research has the intention of assessing the effect of different signal configurations into the pedestrians' start-up time based on pedestrian characteristics, and thereby, find the best signal configurations with minimum start-up delay. Data was obtained from the video footages from three different pedestrian crossings; (1) Traditional red and green phase, (2) Countdown with waiting time phase, (3) Countdown without waiting time phase. One-way ANOVA statical test was used to evaluate whether there exists any significant deference between these signal configurations. Further, Post-Hoc (Tukey) test was conducted to evaluate which specific signal configuration differs significantly from each other. Finally, a multiple linear regression model was developed by considering start-up time as dependent variable and pedestrian characteristics and signal configurations as independent variables. The model was validated with a $R$ value of 0.75 and pedestrian characteristics such as age, gender, having a bag, and the signal configurations are highly significant to the start-up time in the proposed regression model.
\end{abstract}

Keywords: Start-up time; Crossing pedestrians; Different signal configurations; Startup delay

\section{Introduction}

A pedestrian crosswalk is a demarcated place for pedestrians to cross the road safely. Pedestrian crosswalks can be either signalized or non-signalized. However, it is important to design the pedestrian crosswalks as signalized crosswalks to reduce mainly the vehiclepedestrian conflicts. Most of the researchers found that, signalized pedestrian crosswalks can reduce the pedestrian illegal road crossings and reduce the excessive delays for traffic due to the pedestrian crosswalks such as Lipovac et al. [1], Prasanthika et al. [2], Knoblauch et al. [3]. Different signal configurations are designed for such signalized crosswalks. Most frequently available signal configurations in Sri Lanka can be groups as (1) Traditional red and green displays, (2) Countdown with waiting time displays, (3) Countdown without waiting time phase, as shown in Figure 1.
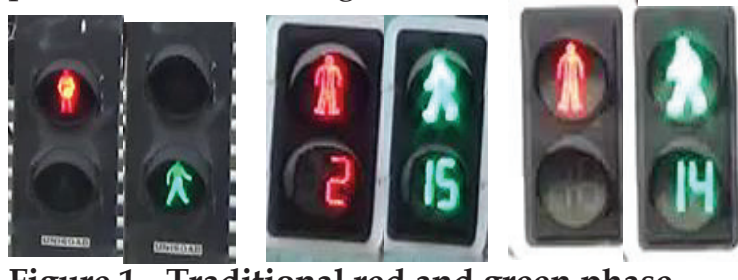

Figure 1 - Traditional red and green phase, Count-down with waiting time phase, Countdown without waiting time phase.
It is observed that pedestrians react differently with signal configuration. Most common reactions of pedestrians are early start-up and delayed start-up. Of course, it is true that many other factors like pedestrian characteristics (age, gender, group size, etc.) and environmental characteristics (sunny and rainy weather) also contribute to those reactions. If pedestrians started to move from the curb during the red light, it is termed as early start-up time whereas if the pedestrians start moving from the curb after the green light, then it is termed as delayed start-up time. Both delays have negative impacts on the safety of pedestrians.

Early start-up time has a negative impact for pedestrian interaction with vehicles and delayed start-up time effects to the crossing time of other pedestrians and possibility of

Eng. (Dr.) V. Wickramasinghe, AMIE(SL)BSc.Eng.(Hons),

MEng, PhD, Senior Lecturer, Sri Lanka Institute of

Information Technology, Email:vasantha.w@sliit.lk

(iD) http://orcid.org/0000-0001-9031-3382

Mr. P.A Wijesinghe, BSc.Eng.(Hons) in Civil Engineering

(Sri Lanka Institute of Information Technology)

Email:pasinduwijesinghe76@gmail.com

(iD http://orcid.org/0000-0003-1426-6304

Miss. K.G.M Lakmali, BSc (Special) in Statistics

(Colombo), Lecturer in Faculty of Humanities and Sciences,

Sri Lanka Institute of Information Technology.

(iD http://orcid.org/0000-0002-2707-9156 
exposing to vehicles during the crossing period. Mark and David. [4] found that most of the procedures only consider the crossing time when designing the signal cycle time in pedestrian crosswalks and crossing time is taken as crosswalk length divided by walking speed. But according to the Manual of Uniform Traffic Control Devices for Streets and Highways (FHWA, 2009), pedestrian crossing time is the summation of the start-up time and the time required to cross the road. Therefore, it is essential to pay attention towards the startup time with different signal configurations. This study has the aim of finding the best signal configuration in order to minimize start-up times. Further, it is to develop a statistical model to estimate the start-up time with different pedestrian characteristics and signal configurations.

There is many literature related to pedestrian behaviour, characteristics, crossing time and safety but only few have considered the pedestrian start-up time and different signal arrangements in their studies. So, paying attention to the start-up time of crossing pedestrians with different signal configurations can improve the pedestrian safety at crosswalks. Jayatilake et al. [5] conducted a series of field studies to estimate the start-up time of crossing pedestrians with different factors (age, gender and group size) and their familiarity with the signal phases. But they considered only one signal configuration for their study. According to the results, they found that these factors have significantly influenced on the start-up time and shows a significant difference of their familiarity with signal phases. Also, Knoblauch et al. [3] collected data to evaluate pedestrian walking speed and pedestrian start-up time with different conditions relative to several factors like age, gender, weather, crosswalk type, signal cycle type, curb height, street width etc. Data was collected on weekdays with the daylight conditions and a period of 8 hours collection time. Lipovac et al. [1] have collected data to evaluate the pedestrian behaviour at signalized pedestrian crossing installations before countdown displays and after installing countdown displays. Analysis of the data was based on the video recorded data and behaviour of pedestrians relative to the factors like with and without countdown displays, different vehicle flow and different locations. The results of the study found that countdown displays reduce the total number of violators, regardless of the location and traffic flow.
Prasanthika et al. [2] have carried out their studies to examine the crossing speed variations of the pedestrians at pedestrian crossings with and without countdown signal phases. Data analysis of this study was based on the video recorded data and they have carried out their studies with both descriptive and statistical analysis. In order to check whether there was a significant difference to the pedestrian speed with and without countdown displays a simple statistical test (Unpaired $\mathrm{t}$ Test) was preformed and they found that there was a significant difference for two samples of the crosswalks. Results of their study showed that average speed of the pedestrians was a higher value at countdown pedestrian crosswalks.

Ma et al. [6] conducted a research to examine the impact of countdown displays on the pedestrian behaviour, and video recording systems were installed at few pedestrian crosswalks to collect the data of pedestrian behaviour in crosswalks. For their data observation process, peak hours of the pedestrian volume on weekdays were selected.

Golani et al. [7] have carried out their study to propose a model for estimating the delay time of pedestrians at signalized crosswalks and collected data by using video graphic technique and observational survey. Also, Fu and Zou [8] studied the effect of installation of the countdown displays in school intersections and analysis was based on the video recording data with different types of pedestrian signals.

The results obtained from these studies show that start-up time is a significant value to be considered, and paying attention to start-up time is an important matter when designing the pedestrian green time for the pedestrian signal phase. In this research, authors have aimed to assess the variations of the start-up time of crossing pedestrians with different signal configurations and pedestrian characteristics to come up with the most suitable pedestrian crossing among the available signal configurations. By conducting this study and the results, interaction between vehicle and pedestrian can be minimized and pedestrian can safely cross the road within the green time phase in a safe signalized crosswalk.

\section{Site Selection and Methodology}

Methodology of this study consists of four major tasks as discussed below. 


\subsection{Site Selection}

Sites were selected according to site characteristics and different signal configurations available in Sri Lankan road system. Since this study mainly considers the pedestrian behaviour in front of the pedestrian crosswalks, it is required to select a suitable place where all the potential pedestrians and site characteristics can be clearly captured. Selected sites were located at a place where a considerable pedestrian volume can be obtained and having a vantage point where pedestrian's feet and curb of the pedestrian crossing can be clearly captured. By considering the above characteristics, three sites were selected and all the selected areas were high commercial areas and $3^{\text {rd }}$ location is in front of an academic institution. Selected locations were identified as four lane, two-way dual carriageways and four lane, two-way single carriageways. Figures 2 to 4 show the selected sites according to the selected signal configurations.

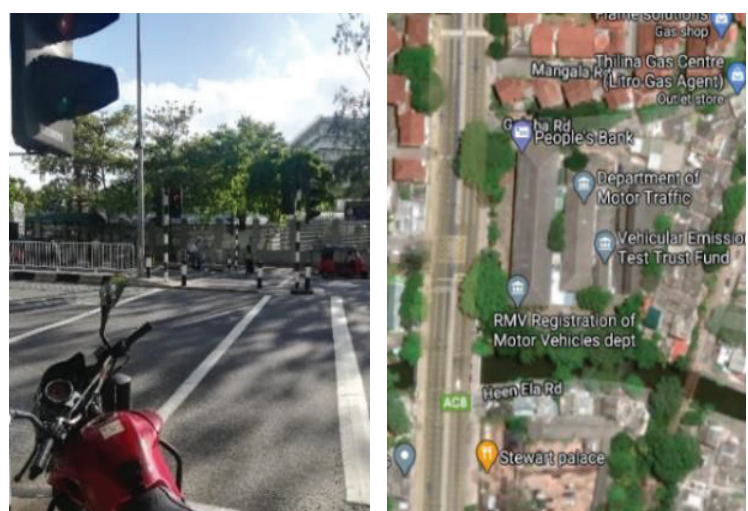

Figure 2 - Traditional Red and Green Signal Phase-In front of Department of Motor

Traffic, Narahenpita $\left(6^{\circ} 53^{\prime} 59.604^{\prime \prime} \mathrm{N}, 79^{\circ} 52^{\prime}\right.$ 38.1792" E)
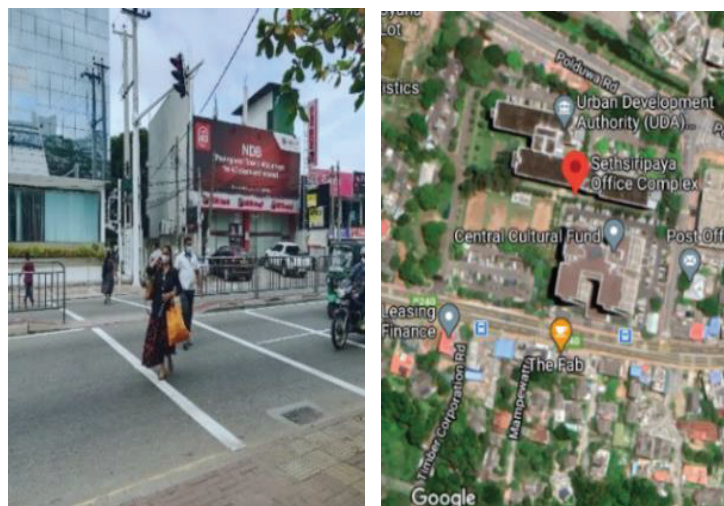

Figure 3 - Count-Down Signal Phase with waiting time- In front of Sethsiripaya Building Complex (654'08.4"N, $\left.9^{\circ} 54^{\prime} 54.6^{\prime \prime} \mathrm{E}\right)$
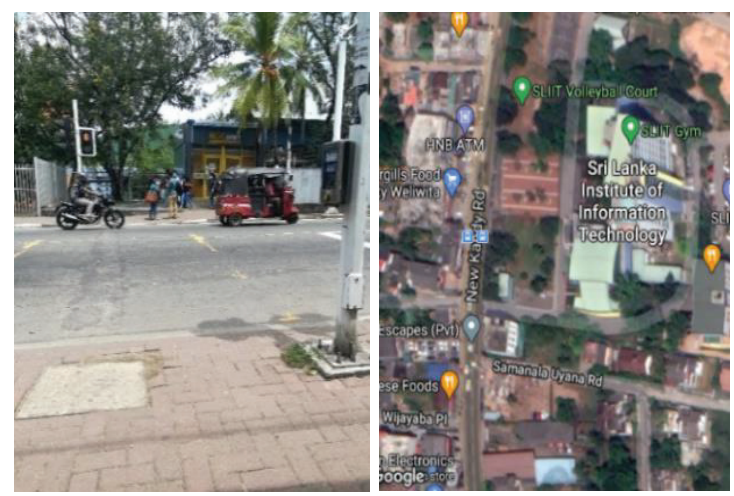

Figure 4 - Count-Down Signal Phase without waiting time- In front of SLIIT Malabe Camps $\left(6^{\circ} 54^{\prime} 51.4836^{\prime \prime} \mathrm{N}, 7^{\circ} 58^{\prime} 19.884^{\prime \prime} \mathrm{E}\right)$

\subsection{Data Collection}

Data collection process was based on the video recording-technique. Digital high-performance cameras were used for this purpose. Cameras were placed using tripods, where pedestrians and signal phases can be clearly captured in each three signal configurations. Data collection was done during the peak hours on weekdays, where high volume of the pedestrians can be identified in each three-signal configuration.

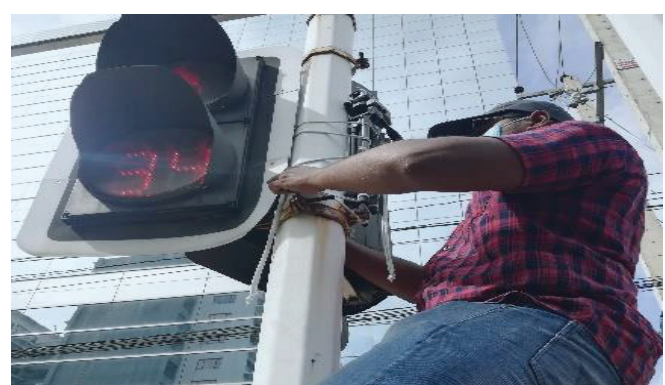

Figure 5 - Fixing Digital Cameras Using a Tripod

\subsection{Data Extraction}

In order to estimate the start-up time of crossing pedestrians, recorded videos at the three places were extracted using a Video to JPG converter. Videos were converted into images with an accuracy of 0.1 seconds and using these images, start-up times of pedestrians were identified.

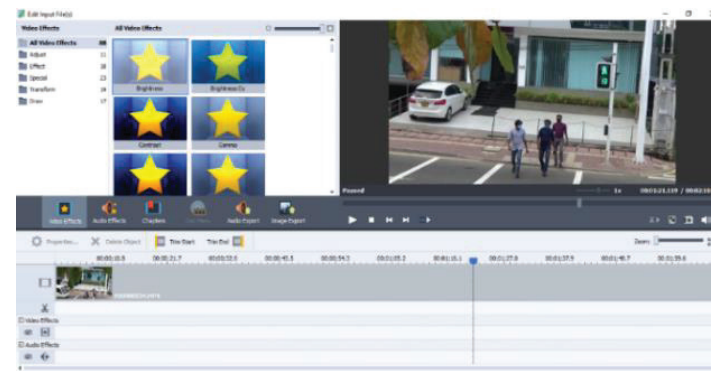

Figure 6 - Data Extraction using AVS-video Converter 
Pedestrians who wait at the curb line only selected as the pedestrian sample and 210 pedestrians from each of these three locations were selected for data analysis. While observing the pedestrian foot movement, the start-up time of each pedestrian was obtained. In order to evaluate the significance of the pedestrians in the start-up time, age, gender, group size and having a bag when crossing the road were observed using these extracted images. In each three signal configurations (1Normal Red \& Green phase, 2-Count-down display with waiting time and 3-Count-down display without waiting time) start-up time with pedestrian characteristics were observed as shown in Table 1.

Table 1 - Pedestrian Characteristics

\begin{tabular}{|l|l|l|}
\hline & $\begin{array}{l}\text { Pedestrian } \\
\text { Characteristics }\end{array}$ & $\%$ of People \\
\hline Age & Less than 30 & $35.7 \%$ \\
& $\begin{array}{l}\text { years } \\
\text { 30<age>60 years } \\
\text { Above 60 years }\end{array}$ & $19 \%$ \\
\hline Gender & Male & $57.2 \%$ \\
& Female & $42.6 \%$ \\
\hline Group Size & Alone or more & $30.2 \%$ \\
& $\begin{array}{l}\text { Two or } \\
\text { (Group) }\end{array}$ \\
\hline $\begin{array}{l}\text { Having a Bag } \\
\text { or Umbrella }\end{array}$ & $\begin{array}{l}\text { Having a bag } \\
\text { Without having } \\
\text { a bag }\end{array}$ & $54.1 \%$ \\
\hline
\end{tabular}

\subsection{Data Analysis}

Under data analysis, both descriptive and advanced statical analysis were conducted. Initially, cumulative frequency diagrams of start-up time (both early and delayed start-up time) were developed. The main objective of this study is to check whether there exists any influence from signal configuration towards the pedestrian start-up time. For that, one-way ANOVA (Analysis of Variance) test was performed. Post-hoc multiple comparison test was conducted to check which specific group(s) were statistically different from each other.

To apply the ANOVA test and post-hoc multiple comparison test, the three samples should be completely independent and normally distributed. To check whether there is any significant difference in between the three signal configurations, the following hypothesis was used,

$$
\begin{array}{ll}
\text { - } & \mathrm{H}_{0} ; \mu_{1}=\mu_{2}=\mu_{3} \\
& {\left[\mu_{\mathrm{i}}-\text { Mean Start-up time for } \mathrm{i}^{\text {th }}\right. \text { signal }} \\
\text { - } & \mathrm{H}_{1}=\text { At least one mean }\left(\mu_{\mathrm{i}}\right) \text { is different }
\end{array}
$$

For checking this relationship, 210 data samples from each signal configuration were used. ANOVA statistical analysis was used to find the significant deference among the three signal configurations.

At the final stage of this study, a multiple linear regression analysis was performed using step wise method by taking start-up time as dependent variable while the pedestrian characteristics and signal configurations as independent variables. Hence, start-up time can be predicted according to the pedestrian characteristics (gender, age, bag and the group size) and signal configurations. Dummy

\begin{tabular}{|c|c|}
\hline $\begin{array}{l}\text { Categorical } \\
\text { Variable }\end{array}$ & \\
\hline Age & $\begin{array}{l}' 0 \text { ' for Less than } 30 \text { yrs } \\
' 1 \text { ' for } 30<\text { age }<60 \\
' 2 \text { ' for more than } 60 \text { yrs }\end{array}$ \\
\hline Gender & $\begin{array}{l}' 1 \text { ' for Male } \\
\text { ' } 0 \text { ' for Female }\end{array}$ \\
\hline Group size & $\begin{array}{l}'{ }^{\prime}{ }^{\prime} \text { for Alone } \\
{ }^{\prime} 0^{\prime} \text { for Group (More than } \\
1 \text { ) }\end{array}$ \\
\hline Having a Bag & $\begin{array}{l}' 1 \text { ' for Having a Bag } \\
\text { '0' for Without Having a } \\
\text { Bag }\end{array}$ \\
\hline
\end{tabular}
variables were created for categorical variables as shown in Table 2.

Table 2 - Description of Categorical Variables

The data sample was randomly divided into two parts called training data $(70 \%$ of total data sample) and test data (30\% of total data sample). Training data was used to develop the model and test data was used to validate the model. To develop the model stepwise method is used and in order to check the significance of independent variables in the obtained model, $\mathrm{p}$ values of the regression coefficients were compared with significance level of 0.05 .

Independent variable is considered as significant to the model if $p$-value of the regression coefficient is less than 0.05 while otherwise, independent variable considered as insignificant to the model. Then, using the test data, fitted model was validated. Finally, the assumptions for this regression model were checked as the model will not be appropriate if underline assumptions are violated.

\section{Results and Discussion}

Extracted data (both early start-up and delayed start-up times) was used to develop a 
cumulative frequency distribution diagram as shown in Figure 7. It is clearly evident that pedestrians behave differently under each signal configuration.

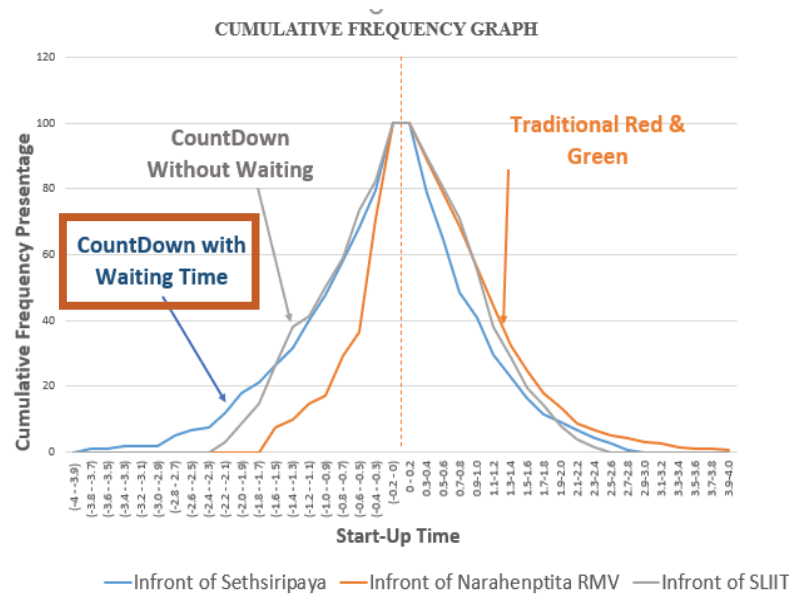

\section{Figure 7 - Cumulative Frequency Percentage for all Three Signal Configurations}

As shown from the cumulative frequency graph, the most effective in terms of minimum start-up pedestrian delay is the count down with waiting time signal configuration.

\subsection{ANOVA Statistical Analysis}

In order to check whether there exists any significant difference among start-up times of these three signal configurations ANOVA test (Analysis of variance) was performed. Table 3 shows the ANOVA test results. According to the ANOVA results, $p$ value $=0.000<0.05$ indicates that null hypotheses of all group mean are equal and rejected at 5\% level of significance. It implies that there exists at least one signal configuration which is different from others at $5 \%$ level of significance.

Table 3 - One-way ANOVA Test Results

\begin{tabular}{|c|c|c|c|c|c|}
\hline & $\begin{array}{c}\text { Sum of } \\
\text { Squares }\end{array}$ & df & $\begin{array}{c}\text { Mean } \\
\text { Square }\end{array}$ & F & Sig \\
\hline $\begin{array}{c}\text { Between } \\
\text { Groups }\end{array}$ & 90.795 & 2 & 45.398 & $\begin{array}{c}41.0 \\
29\end{array}$ & 0.00 \\
\hline $\begin{array}{c}\text { Within } \\
\text { Groups }\end{array}$ & 690.444 & 624 & 1.106 & & \\
\hline Total & 781.239 & 626 & & & \\
\hline
\end{tabular}

One-way ANOVA cannot describe which specific groups were significantly different from each other. For this purpose, post hoc test (Tukey) was conducted and Table 4 shows the post hoc test results. From this method, the means of three different samples can be compared as pair of sample means using their absolute differences. Results obtained from the post hoc test show that all the three signal configurations are significantly different from each other at \%5 level of significance as all $p$ values are less than 0.05 .

Table 4 - Post Hoc Test Results (Tukey)

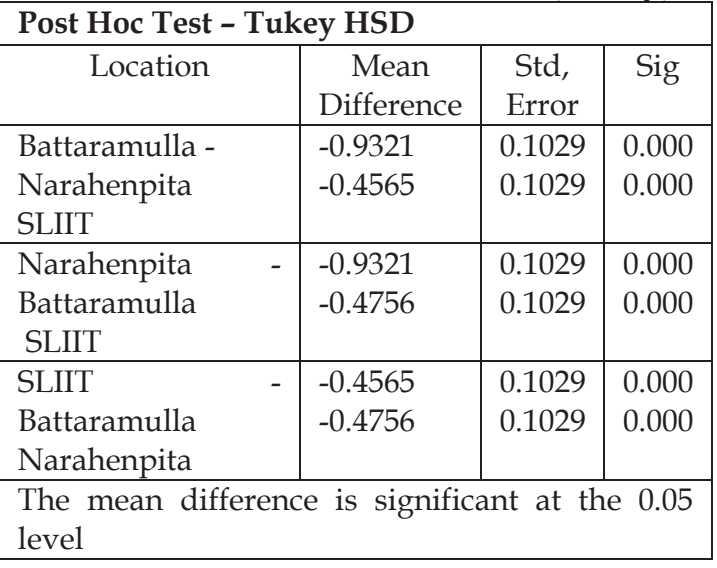

According to the ANOVA, post hoc test results and cumulative frequency graph, it can be concluded that there exists a significant difference in each of these signal configurations and the most effective in terms of minimum start-up delay is given from the count down with waiting time signal configuration.

\subsection{Check for Assumptions of ANOVA Test}

The results of ANOVA test is valid only if they satisfy underlined five main assumptions.

- Assumption\#1

Dependent variable should be measured at ratio scale or interval scale. In this study, dependent variable is in ratio scale.

- Assumption\#2

There should be at least two levels in categorical variable (two or more groups). In this study, there are three levels in categorical variable (Three groups).

- Assumption\#3

Independent observations- Population groups should be independent with each other. In this study data was from three different locations and three different signal configurations.

- Assumption\#4

Dependent variable should be normally distributed. In this study, normality of the dependent variable has been checked using histogram of the dependent variable as below. According to Figure 8, it can be seen that dependent variable is normally distributed. 


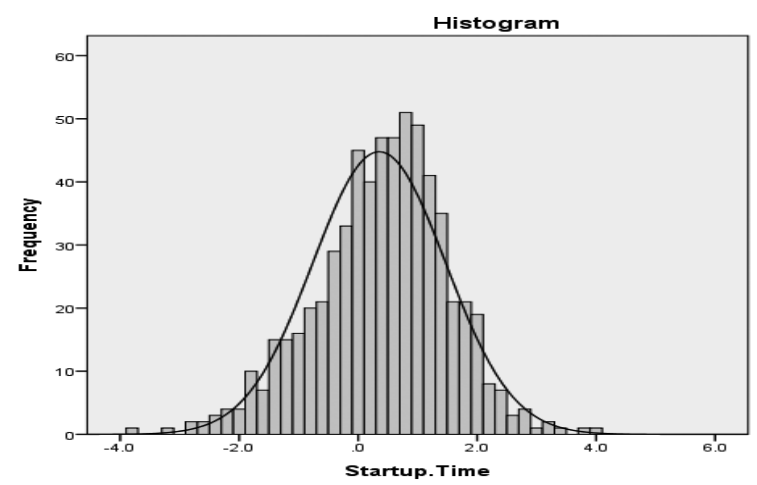

Figure 8 - Histogram for Start-up Time

\section{- Assumption\#5}

Homogeneity of variance among groups In this study a test was carried out to check the equality of variances. The $\mathrm{p}$-value of the test was 0.08 which is greater than 0.05 , thus it does not reject the null hypothesis, implying that of all three groups have same variance. This concludes that the assumption of homogeneity of variance among groups have been satisfied at 5\% level of significance.

\subsection{Multiple Linear Regression Analysis}

According to the results, p-value of the fitted model is 2.2e-16 which is less than 0.05. Thus, we have enough evidence to suggest that the fitted model is significant at 5\% level of significance with independent variables; Gender, Age, With or without Bag and the signal-type. According to the results, equation of the fitted model is as shown is equation (1),

\section{Table 5 - Table of Coefficients}

\begin{tabular}{|l|c|c|c|}
\hline Coefficients: & Estimate & $P$ value & $\mathrm{t}$ value \\
\hline Intercept & -1.02112 & $1.54 \mathrm{e}^{-15}$ & -8.281 \\
\hline Gender - Male & 0.41052 & $3.27 \mathrm{e}^{-5}$ & 4.198 \\
\hline Age- 30<>60 & 0.60516 & $4.56 \mathrm{e}^{-8}$ & 5.567 \\
\hline Age- Above 60 & 1.24534 & $4.73 \mathrm{e}^{-14}$ & 7.798 \\
\hline Bag- With Bag & 0.27358 & 0.00671 & 2.724 \\
\hline $\begin{array}{l}\text { Traditional red } \\
\text { and green Phase }\end{array}$ & 0.88575 & $2.46 \mathrm{e}^{-13}$ & 7.558 \\
\hline $\begin{array}{l}\text { Count down } \\
\text { with-out waiting } \\
\text { time phase }\end{array}$ & 0.82337 & $3.38 \mathrm{e}^{-10}$ & 6.430 \\
\hline \\
Start-up = 0.41052(Male) + 0.60516(Age 30- 60) \\
Time + 1.24534(Age above 60) + 0.27358( \\
With bag) + 0.88575 (Traditional red \\
and green) + 0.82337(Count down \\
without waiting time) - 1.02112
\end{tabular}

The estimated values for the equation fitted by the analysis shows that, there is a positive value if the crossing pedestrian is a male, age above 30, carrying a bag with the person and when he/she crosses the road in a red and green phase and countdown without waiting time phase.

\subsection{Check for Assumptions}

Model validation is done to check whether model assumptions are violating and to ensure that predicted values from the fitted model are much closer to actual values.

\section{- Assumption\#1}

Dependent Variable should be measured at the continuous level. As the start-up time is measured in an ordinal scale, this is satisfied.

\section{- Assumption\#2}

This was checked using Durbin-Watson test and the value should be between 1.5-2.5. During this study, DW $=1.5309$ and the value got satisfied.

\section{- Assumption\#3}

Data needs to show homoscedasticity- This was checked using scatter plot of standardized residuals vs fitted values. According to Figure 9, since there is no particular pattern in the plot, it concludes that the assumption of homoscedasticity has been satisfied.

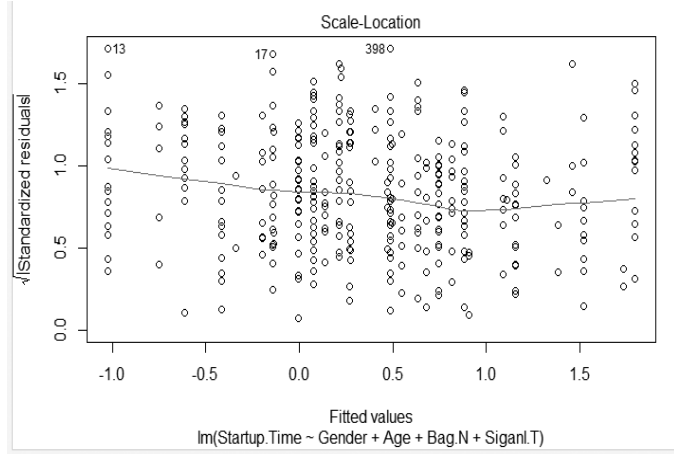

Figure 9 - Standardized Residuals vs Fitted Values

\section{- Assumption\#4}

Residuals should be normally distributed. This was checked using normal Q-Q plot of residuals and Anderson Darling test.

As shown in Figure 10, it can be seen that all the observations tidily lie around the line. Moreover, value obtained from Anderson Darling test was 0.49654 along with a $p$ value of 0.2118 which is greater than 0.05 . This concludes that residuals are normally distributed. 


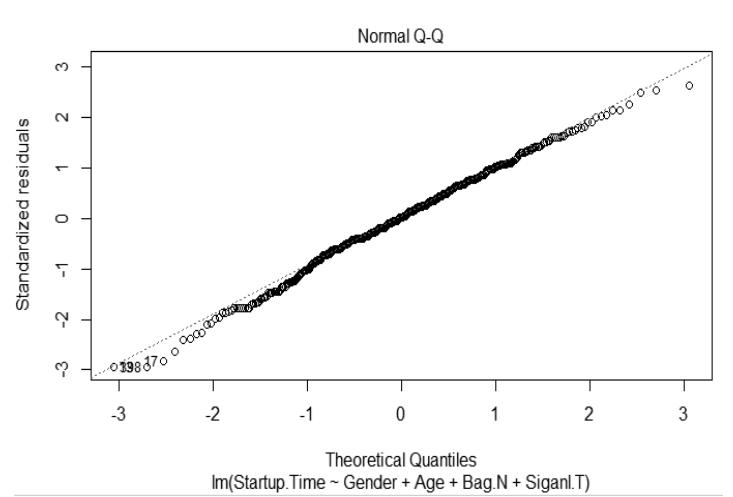

Figure 10 - Normal Q-Q Plot

Normality test - The value obtained was 0.49654 along with a p-value of $0.2118>0.05$. (Satisfied)

\section{- Assumption\#5}

There should be a linear relationship in between dependent variables and independent variables. This can also be checked using Durbin Watson test. Since $\mathrm{DW}=1.5309$, linearity assumption is also satisfied. According to these results, there are no violations in model assumptions. Finally, the correlation between the actual data and the predictable data was checked and a correlation coefficient of 0.50 was obtained for the model, which is an acceptable value for practically tested data.

\section{Conclusion}

Currently, different types of pedestrian signal configurations are available in Sri Lankan road system. The most common pedestrian signal configurations are in three types (1) traditional red and green displays, (2) countdown with waiting time displays, (3) countdown without waiting time. This research was conducted to find the best signal configuration in terms of minimizing start-up time of pedestrians. From the cumulative frequency distribution diagram, it clearly showed the variations of both early and delayed start-up times with different signal configurations. In order to check these differences statistically, one-way ANOVA test and the Post-Hoc multiple comparison test were conducted and from the test results it was evident that there exists a significant difference among three signal configurations. Count down with waiting time was the most effective and has the minimum Start-up delay in the pedestrian's crossing time. From this signal configuration, interaction between the vehicles and pedestrians at the crosswalks can be minimized and the delay due to the pedestrian crossing into main stream can be reduced.
Hence the countdown signal configurations can improve more effectively with using the startup time consideration.

Further, a multiple linear regression model was fitted using start-up time as the dependent variable. Signal configuration and pedestrian characteristics such as age, gender, group size, carrying bags or not, were considered as independent variables. The analysis shows that the most significant variables in deciding the start-up time are signal configuration and pedestrian characteristics such as age, gender and with or without bag. However, this research was conducted during peak hours in urban areas and the findings of this research could be useful for traffic planning work whilst assuring the safety of both pedestrians and drivers in a more effective way during peak hours.

\section{References}

1. Lipovac, K., Vujanic, M., Maric, B. and Nesic, M. "The Influence of a Pedestrian Countdown Display on Pedestrian Behavior at Signalized Pedestrian Crossings," Transportation Research Part F, 2003, pp. 121-134.

2. Madushi, P., Ranasinghe, N., Karunarathne, T., Madushani, H. and Wickramasinghe, V. "Case Study on Comparison of Crossing Speed Variation of Pedestrians at Signalized Crosswalks with and without Countdown Displays," 13th International Conference of Eastern Asia Society for Transportation Studies. (EASTS), Volume 12, 2019.

3. Knoblauch, R. L., Pietrucha, M. and Nitzburg, M. "Field Studies of Pedestrians Walking Speed and Start-up Time," Transportation Research Record 1538, Journal of the Transportation Research Borad, National Research Council, Washington. D.C, pp. 27- 38 .

4. Virkler, M. R. and Guell, D. L. 1984. "Pedestrian Crossing-Time Requirements at Intersections", Transportation Research Record, National Research Council, National Academy of Sciences USA, 959, pp. 47-51.

5. Jayatilake, S., Premachandra, N., Wickramasinghe, V. and Dissanayake., N., "Affect of Different Factors on the Start-up Times of Pedestrians," $12^{\text {th }}$ International Conference of Eastern Asia Society for Transportation Studies, Vol.11,2017, pp. 18-21.

6. Ma, W., Liao, D. and Bai, Y., “Empirical Analysis of Countdown Signals on Pedestrian Behavior", Proceedings of the Institution of Civil Engineers Transport 168, February 2015, pp. 15-22. 
7. Golani, A. \& Damti, H, “Model for Estimating Crossing Times at High Occupancy Crosswalks", "Transportation Research Record No. 2002, Journal of the Transportation Research Board, National Academies, Washington. D.C, 2007, pp. 125130.

8. Lianning $\mathrm{Fu}$, Nan Zou, "The Influence of Pedestrian Countdown Signals on Children's Crossing Behaviour at School Intersections," Accident Analysis and Prevention 94, 2016, pp. 73-79. 